\title{
Exact solution for the response of an arbitrarily-curved beam subject to a moving load
}

\author{
L. Emir Sakman ${ }^{1 *}$ \\ ${ }^{1 x}$ Istanbul University-Cerrahpaşa Department of Mechanical Engineering, İstanbul Turkey, (ORCID: 0000-0002-9599-8875), sakman@iuc.edu.tr
}

(International Conference on Design, Research and Development (RDCONF) 2021 - 15-18 December 2021)

(DOI: 10.31590/ejosat.1048496)

ATIF/REFERENCE: Sakman, L. E. (2021). Exact solution for the response of an arbitrarily-curved beam subject to a moving load. Avrupa Bilim ve Teknoloji Dergisi, (32), 414-423

\begin{abstract}
An arbitrarily curved beam under the effect of a moving load has been considered. An analytical series solution has been developed for the case when the beam cross-section is symmetrical so that it resides in a plane during the motion. The moving force is assumed to be a singular force sliding through the length of the beam with constant speed while its direction always pointing in the principal normal of the curved shape of the beam. After developing the general solution for any plane beam, example computations were carried out on a specific example by means of power series expansion.
\end{abstract}

Keywords: Arbitrarily-curved, Moving load, Power series.

\section{Hareketli bir yüke maruz kalan keyfi kavisli bir kirişin tepkisi için kesin çözüm}

$\ddot{O} z$

Rastgele bir düzlem eğimli kirişin hareketli bir yüke tepkisi analitik olarak incelenmiştir. Kiriş kesiti belirli bir simetriye sahip olacak şekilde sınırlandırılmıştır, böylece kiriş hareket eden kuvvetin etkisi altındayken düzleminde kalır. Hareket eden kuvvetin, yönü daima kirişin kavisli şeklinin ana normalini işaret ederken, kirişin uzunluğu boyunca sabit hızla kayan tekil bir kuvvet olduğu varsayılır. Genel denklemler herhangi bir düzlem eğrisi için verilmiş ve özel bir örnek için kuvvet serileri yöntemi kullanılarak çözülmüştür.

Anahtar Kelimeler: Keyfi kavisli, Hareketli yük, Kuvvet serileri.

\footnotetext{
* Corresponding Author: sakman@iuc.edu.tr
} 


\section{Introduction}

While many of the curved elements used in engineering structures are very common shapes like circular arcs and helixes, arbitrarily shaped elements also find usage which may be expected to expand with the ever increasing complexity of mechanisms, and aerospace and civil structures (bridges etc.). The general equations for the small vibrations of an arbitrarilyshaped space beam were derived long ago; the definitive reference for this (and many other problems) is (Love, 1944) according to whom the general formulation for arbitrarilycurved space beams is due to Clebsch (1895).

Most of the studies presented in the literature related to moving loads and curved beams involve circular arcs and numerical methods. We cite the following recent studies: (Wu and Chiang, 2004) computed the response of a circular arc to a moving load by using the finite element method. (Gulyayev and Tolbatov, 2004) considered a liquid mass moving inside a helicoidal tube. (Wayou et al., 2004) investigated the nonlinear dynamics of a straight beam due to a moving load. (Forbes and Randall, 2004) solved the case of a full circular ring under the effect of a moving load using the mode expansion method. (Huang et al., 2011) solved the nonlinear vibrations of a curved beam which is excited by an outside agent; in this case the agent was not a moving load, nevertheless the mathematical formulation is very similar.

The number of studies about moving loads on curved beams seems to be scarce. Since the solution procedures for these types of problems involve computation of natural frequencies and mode shapes, we mention some of the related work. (Tufekci and Dogruer, 2006) gave and exact solution for the out of plane vibrations of a circular arc including rotatory inertia and shear effects. (Yang et al., 2008) investigated free in plane vibrations of arbitrary curved beams including the effects of extensibility, shear and rotatory inertia using finite element formulation. (Ozturk, 2011) investigated the in-plane vibrations of a pre-stressed curved beam obtained from a larger straight beam by using finite elements. (Tolomeo, 2005) investigated a method for forming parabolically shaped beams to optical accuracy by using the curved beam theory. (Lin and Hsieh, 2007) gave closed form solutions for laminated curved beams. (Piovan and Cortinez, 2007) investigated thin-walled composite curved beams including shear effects using finite elements. (Wang and Liu, 2013) used elasticity theory solutions (Airy stress function) for anisotropic, functionally

\section{Material and Method}

The curved beam axis, which is the curve formed by the centroids of the cross-sections, will be named the beam axis. Let the tangent to beam axis at any point along the beam be the $\mathrm{z}$-axis. $\mathrm{y}$-axis is normal to the plane in which the beam resides whe not moving; and $\mathrm{x}$-axis, which is the normal to beam axis within the plane, completes the orthogonal frame. If the $\mathrm{xz}$ plane is a symmetry plane of the beam axis, the beam would not move outside of the plane unless forced in that direction. Therefore, the moving load will be assumed to be in $\mathrm{x}$-direction.

The derivation of the governing equations were given by (Love, 1944) using equation of motion. The derivation is summarized graded, curved beams. (Lenci and Clementi, 2009) developed a simple mechanical model for space curved beams including bending and torsion effects. (Lim et al., 1997) investigated the Euler-Bernoulli and Timoshenko models comparatively for a curved beam of constant curvature. (Luu et al., 2015) used a finite element approach to investigate laminated curved beams including extensibility, shear and rotatory inertia effects. (Tseng et al., 2000) studied vibrations of composite laminated curved beams including a Timoshenko beam model. (Krishnan and Suresh, 1998) developed cubic elements for analysis of curved beams. (Kim et al., 2003) investigated free vibrations of thin-walled, non-symmetric, curved beams including second order effects. (Lee et al., 2008) studied out of plane vibrations of plane curved beams using numerical solutions of the governing differential equations by Runge-Kutta methods. (Huang et al., 2000) did the same as (Lee et al., 2008) but using a finite element approach and arbitrary cross section. (Huang et al., 1998) studied in plane vibrations of curved beams using power series approach.

Classical curved beam problem seems to be an active research area where many methods of applied mathematics still can be tested and applied.

This paper will consider an arbitrarily curved EulerBernoulli beam. To appreciate the effects of arbitrary curvature, the internal structure of the beam will be taken as simple as possible. The beam is loaded with a singular force along its normal direction, while the load moves through the length of the beam with a constant velocity. The end result is a sixth order partial differential equation with variable coefficients which turns out to be non-self-adjoint; therefore, the mode-shapes do not constitute an orthogonal family. When the time-response is computed, this leads to an infinite system of coupled ordinary differential equations which is truncated to get an approximate series solution.

As a concrete example in which the curvature changes along the beam, a specific shape of the beam is chosen such that the form of the resulting ordinary differential equation for the mode shapes is amenable to power series solution. This results in an analytical (series) solution. More general beam shapes would undoubtedly require some type of numerical approximations to be made.

using vectorial notation in the general 3-dimensional case (Sakman and Uzal, 2017).

The beam described here is a conservative system; thus the governing differential equations should result from the Hamilton's principle,

$$
\delta \int_{t_{1}}^{t_{2}} L=0
$$


where

$$
L=T-V
$$

is the Lagrangian with $\mathrm{T}$ representing kinetic energy and $\mathrm{V}$ representing potential energy of the system. We show the components of moment as $M_{x}, M_{y}, M_{z}$ and the components of body force as $f_{x}, f_{y}, f_{z}$. The beam will not move out of its plane if

$$
M_{x}=M_{z}=0, \quad f_{y}=0
$$

Kinetic and potential energies can be expressed as,

$$
\begin{gathered}
T=\frac{1}{2} \int_{0}^{s} m\left(\dot{U}^{2}+\dot{W}^{2}\right) d s \\
V=\frac{1}{2} \int_{0}^{s}\left(M_{y}\left(\kappa_{y}-\kappa_{\mathrm{y} 0}\right)\right) d s
\end{gathered}
$$

dot showing differentiation with respect to time. In Eqs. (3) and (4), s and $\kappa_{y}, \kappa_{\mathrm{y} 0}$ are the arc-length and curvature after and before the deformation, $\mathrm{m}$ is the mass per unit length, $\mathrm{U}, \mathrm{W}$ are deformations along $\mathrm{x}$ and $\mathrm{z}$-axes. The curvatures before and after deformation are related by

$$
\kappa_{y}=\kappa_{y 0}+\frac{\partial}{\partial s}\left(\frac{\partial U}{\partial s}+\kappa_{y 0} W\right)
$$

During deformation, the beam axis is assumed to not undergo extension; this is expressed as

$$
\frac{\partial W}{\partial s}-\kappa_{y 0} U=0
$$

The relation between the bending moment and curvature change is expressed as

$$
M_{y}=b\left(\kappa_{y}-\kappa_{y 0}\right)
$$

where

$$
b=E I_{y}
$$

is the bending rigidity. $\mathrm{E}$ is the the elastic modulus of the beam material, $I_{y}$ is the second moment of area of the cross section around y-axis.

In the linearized theory (small deformation) the curvatures appearing in Eq. (4) can be assumed to be the curvatures before the deformation. The deformation component $U$ can be eliminated by (6), $U=\left(\frac{1}{\kappa_{y 0}}\right) \frac{\partial W}{\partial s}$ and the variational problem (1) can be expressed in terms of W only. Then, the EulerLagrange equations of (1) gives

$$
G(w)+\frac{\kappa_{0}^{\prime}}{\kappa_{0}^{2}} f_{x}-\frac{1}{\kappa_{0}} f_{x}^{\prime}+f_{z}=m\left(\frac{\partial^{2} w}{\partial t^{2}}+\frac{2 \kappa_{0}}{\kappa_{0}^{2}} \frac{\partial^{3} w}{\partial t^{2} \partial s}-\frac{1}{\kappa_{0}^{2}} \frac{\partial^{4} w}{\partial t^{2} \partial s^{2}}\right)
$$

where $G(w)$ is a linear sixth order partial differential operator. It involves only differentiation with respect to $\mathrm{s}$ and has variable coefficients also depending on $\mathrm{s}$.
The equations developed here are completely general. As a concrete example, we consider a beam for which the curvature before the deformation is given as

$$
\kappa_{y 0}=\frac{1}{L \theta}
$$

where we introduced characteristic length $\mathrm{L}$, since the curvature has dimension of inverse length. $\theta$ is the tangential angle which is related to arc-length by

$$
d s=\frac{1}{\kappa_{0}} d \theta
$$

The actual shape of the curve can be found by integrating Eq.(11) and is shown in Figs. 1-10 together with mode shapes.

We assume the moving load with magnitude $\mathrm{F}$ is always in the normal direction of the beam axis and moves with constant velocity $\mathrm{U}$, i.e.,

$$
f_{z}=0, \quad f_{x}=F \delta(s-U t)
$$

where $\delta$ is the Dirac delta function. From Eq. (10) and Eq. (11), the relation between $\mathrm{s}$ and $\theta$ is

$$
S=\frac{L}{2}\left(\theta^{2}-\frac{\pi^{2}}{16}\right)
$$

where $\theta$ changes in the interval

$$
\frac{\pi}{4} \leq \theta \leq \frac{3 \pi}{4}
$$

so that the total length of the beam is $L \pi^{2} / 4$.

The displacement $\mathrm{w}$ is non-dimensionalized by the characteristic length $\mathrm{L}$ and time is non-dimensionalized by $\sqrt{\mu L^{4} / b}$; then $2 U \sqrt{\mu L^{2} / b}$ is a non-dimensional velocity. A suitable non-dimensional force is $2 F L^{2} / b$. For specific computations later we take $L=1$.

Consequently, Eq. (9) becomes,

$$
H(w)+\theta^{7} f_{x}+\theta^{8} \frac{\partial f_{x}}{\partial \theta}=\theta^{8} \frac{\partial^{2} w}{\partial t^{2}}+\theta^{7} \frac{\partial^{3} w}{\partial t^{2} \partial \theta}-\quad \theta^{8} \frac{\partial^{4} w}{\partial t^{2} \partial \theta^{2}}
$$

In Eq. (15) all quantities are non-dimensional, and we defined

$$
\begin{gathered}
H(w)=3\left(30+\theta^{2}\right) w-3 \theta\left(30+\theta^{2}\right) \frac{\partial w}{\partial \theta}+\left(\theta^{4}+42 \theta^{2}+\right. \\
90) \frac{\partial^{2} w}{\partial \theta^{2}}+\theta\left(\theta^{3}-12 \theta^{2}+39 \theta-\right.
\end{gathered}
$$

which is the same as $G(w)$ except that expressed for this specific example and new non-dimensional variables. This simplification (and many others to be encountered later) was carried out by using symbolic manipulation codes. The force term is

$$
f_{x}=F \delta\left(\theta^{2}-\alpha^{2}\right)
$$

with 
European Journal of Science and Technology

$$
\alpha=\sqrt{U t-\frac{\pi^{2}}{16}}
$$

Note that $\alpha$ depends on t. In Eq. (17), the right hand side is simplified by using the delta-function identity

$$
\delta\left(\theta^{2}-\alpha^{2}\right)=\frac{1}{2|\alpha|}[\delta(\theta+\alpha)+\delta(\theta-\alpha)]
$$

As to the boundary conditions we assume that both ends of the beam are fixed, thus

$$
w=0, u=\frac{\partial u}{\partial s}=0
$$

at both ends, which, in terms of these variables become,

\section{Solution}

As the first step for evaluating the response of the beam, we must evaluate the eigensolutions or mode shapes of the nonforced beam. Considering the non-forced or homogeneous form of Eq. (16):

$$
H(w)=\theta^{8} \frac{\partial^{2} w}{\partial t^{2}}+\theta^{7} \frac{\partial^{3} w}{\partial t^{2} \partial s}-\theta^{8} \frac{\partial^{4} w}{\partial t^{2} \partial s^{2}}
$$

with homogeneous boundary conditions Eq. (21), assume time-harmonic solution in the form

$$
w(\theta, t)=e^{i \omega t} W(\theta)
$$

Then Eq. (23) becomes, with prime denoting differentiation with respect to $\theta$,

$$
H(W)=-\omega^{2}\left(\theta^{8} W+\theta^{7} W^{\prime}-\theta^{8} W^{\prime \prime}\right)
$$

with the boundary conditions

$W=W^{\prime}=W^{\prime \prime}=0$ for

$$
\theta=\frac{\pi}{4} \text { and } \frac{3 \pi}{4}
$$

Eq. (25) is an eigenvalue problem with a discrete spectrum. The eigenvalues and eigenfunctions will be shown as

$$
\omega_{n}, \varphi_{n}(\theta), n=1,2,3, \ldots
$$

Leaving aside, for the moment, the solution of the eigenvalue problem, the solution of the forced (non-homogeneous) differential equation can be written as

$$
w(\theta, t)=\sum_{n=1}^{\infty} q_{n}(t) \varphi_{n}(\theta)
$$

The force terms in Eq. (15) are also expanded in the $\varphi_{n}$-basis. For example, the derivative of Dirac delta is expanded as

$$
\delta^{\prime}(\theta-\alpha)=\sum_{n=1}^{\infty} c_{n}(t) \varphi_{n}(\theta)
$$

and the coefficient functions $c_{n}(t)$ are evaluated by multiplying this equation with $\varphi_{m}(\theta)$ and integrating

$$
\left(\varphi_{m}(\theta), \delta^{\prime}(\theta-\alpha)\right)=\sum_{n=1}^{\infty} c_{n}(t)\left(\varphi_{m}(\theta), \varphi_{n}(\theta)\right)
$$

$$
\begin{aligned}
& w=\frac{\partial w}{\partial \theta}=\frac{\partial^{2} w}{\partial \theta^{2}}=0, \text { for } \\
& \theta=\frac{\pi}{4} \text { and } \frac{3 \pi}{4}
\end{aligned}
$$

Some discussion about the boundary conditions will be suitable at this point. The model being developed takes into account motion in the direction of the beam and normal direction in the plane of the beam. The boundary conditions should reflect motion in both directions. Since small displacement magnitudes are assumed, fixed boundary conditions are choosen in the present study for all directions.

We assume that the beam is unloaded at the beginning so that the initial conditions are

$$
w=\frac{\partial w}{\partial t}=0, \text { for } t=0
$$

where the paranthese show the "inner product"

$$
\left(\varphi_{m}, \varphi_{n}\right)=\int_{\pi / 4}^{3 \pi / 4} \varphi_{m}(\theta) \varphi_{n}(\theta) d \theta
$$

Left hand side of (29) is $-\varphi_{m}{ }^{\prime}(\alpha)$ using the ususal properties of delta function, but the right hand side does notlead to a simple expression since $\varphi_{n}(\theta)$ is not an orthogonal family. Therefore, we truncate the infinite series at an iteratively chosen upper limit value $n=N$ and compute the $c_{n}(t)$ coefficients by solving a linear algebraic system of $\mathrm{N}$ equations. Note that $\delta(\theta+\alpha)$ and $\delta^{\prime}(\theta+\alpha)$ do not contribute since $\theta=-\alpha$ is outside the interval.

As a result Eq. (15) becomes

$$
\begin{gathered}
-\sum_{n=1}^{\infty} \omega_{n}^{2}\left(\theta^{8} \varphi_{n}+\theta^{7} \varphi_{n}^{\prime}-\theta^{8} \varphi_{n}^{\prime \prime}\right) q_{n}(t)+ \\
\sum_{n=1}^{\infty} F_{n}(\theta, t) \varphi_{n}=\sum_{n=1}^{\infty}\left(\theta^{8} \varphi_{n}+\theta^{7} \varphi_{n}^{\prime}-\quad \theta^{8} \varphi_{n}^{\prime \prime}\right) \frac{d^{2} q_{n}}{d t^{2}}
\end{gathered}
$$

since $\varphi_{n}$ satisfies Eq. (25a). Here, all terms related to the forcing $\theta^{7} f_{x}+\theta^{8} f_{x}^{\prime}$ have been collected in the middle sum in Eq. (31) in the function $F_{n}(\theta, t)$. Scalar multiplying Eq. (31) by $\varphi_{m}(\theta)$ leads to an infinite system of linear ordinary differential equations with constant coefficients for $q_{n}(t)$, which we similarly truncate at $n=N$,

$$
\begin{gathered}
\sum_{n=1}^{N} \omega_{n}^{2} A_{n m} \frac{d^{2} q_{n}(t)}{d t^{2}}=-\sum_{n=1}^{N} \omega_{n}^{2} A_{n m} q_{n}(t)+\sum_{n=1}^{N} F_{n m}(t) \\
, 1 \leq m \leq N
\end{gathered}
$$

where

$$
\begin{gathered}
A_{n m}=\left(\theta^{8} \varphi_{n}+\theta^{7} \varphi_{n}^{\prime}-\theta^{8} \varphi_{n}^{\prime \prime}, \varphi_{m}\right) \\
F_{n m}(t)=\left(F_{n}, \varphi_{m}\right)
\end{gathered}
$$

Forcing term is the last sum in Eq. (32). Homogeneous system corresponding to Eq. (32) is

$$
\begin{aligned}
& \sum_{n=1}^{N} \omega_{n}^{2} A_{n m} \frac{d^{2} q_{n}(t)}{d t^{2}}=-\sum_{n=1}^{N} \omega_{n}^{2} A_{n m} q_{n}(t), \\
& 1 \leq m \leq N
\end{aligned}
$$

which will have solutions of the form 


$$
q_{n}(t)=Q_{n} e^{r t}
$$

Substituting, we obtain

$$
\begin{aligned}
& \sum_{n=1}^{N} \omega_{n}^{2} A_{n m}\left(r^{2}+\delta_{n m}\right) Q_{n}=0, \\
& 1 \leq m \leq N
\end{aligned}
$$

where $\delta_{n m}$ is the Kronecker delta symbol. Eq. (36) is an algebraic, linear, homogeneous system of equations. Eigenvalues $\left(r_{k}^{2}\right)$ and eigenvectors $\left(Q_{n}^{(k)}\right)$ are evaluated by utilizing the coefficient matrix as usual. In our case all eigenvalues turned out to be distinct,

$$
r_{k}^{2} \rightarrow Q_{1}^{(k)}, Q_{2}^{(k)}, \ldots, Q_{N}^{(k)} ; 1 \leq k \leq N
$$

Then the general solution of the homogeneous system van be written as

$$
\begin{aligned}
& q_{n}(t)=\sum_{k=0}^{N} Q_{n}^{(k)}\left(D_{n}^{(k)} e^{r_{k} t}+E_{n}^{(k)} e^{-r_{k} t}\right) \\
& 1 \leq n \leq N
\end{aligned}
$$

where $D_{n}^{(k)}, E_{n}^{(k)}$ are arbitrary constants. The solution of the nonhomogeneous Eq. (32) is assumed to be of the form

$$
q_{n}(t)=\sum_{N=0}^{N} Q_{n}^{(k)}\left[D_{n}^{(k)}(t) e^{r_{k} t}+E_{n}^{(k)}(t) e^{-r_{k} t}\right]_{(39)} \quad 1 \leq n \leq
$$

by using the variation of constants method: here now $D_{n}^{(k)}(t)$, $E_{n}^{(k)}(t)$ are unknown functions. This leads to two sets of $\mathrm{N}$ equations each

$$
\sum_{k=0}^{N} Q_{n}^{(k)}\left[\frac{d D_{n}^{(k)}}{d t} e^{r_{k} t}+\frac{d E_{n}^{(k)}}{d t} e^{-r_{k} t}\right]=0 \quad, \quad 1 \leq n \leq N
$$

$$
\sum_{n=1}^{N} \sum_{k=0}^{N} \omega_{n}^{2} A_{n m} r_{k} Q_{n}^{(k)}\left[\frac{d D_{n}^{(k)}}{d t} e^{r_{k} t}-\frac{d E_{n}^{(k)}}{d t} e^{-r_{k} t}\right]=
$$$$
\sum_{n=1}^{N} F_{n m}(t), 1 \leq m \leq N \quad \text { (41) }
$$

These are solved for $\frac{d D_{n}^{(k)}}{d t}$ and $\frac{d E_{n}^{(k)}}{d t}$ and integrated to complete the solution.

We still need to deal with the eigenvalue problem consisting of Eq. (25a) and boundary conditions (25b). This can conveniently be done by using the method of power series. Assume an analytic solution around the middle of the range of $\theta$,

$$
W(\theta)=\sum_{n=0}^{\infty} a_{n}(\theta-\pi / 2)^{n}
$$

Substituting in Eq. (25a) after evaluating various derivatives of Eq. (42) leads to a recurrence relation for the coefficients $a_{n}$. The first six coefficients can not be evaluated since there are six boundary conditions to be satisfied. Therefore, the general solution is of the form

$$
W(\theta)=\sum_{k=0}^{5} a_{k} \psi_{k}(\omega, \theta)
$$

where $\psi_{k}(\omega, \theta)$ are power series in $\theta$. Some representative terms resulting from the recurrence relation are shown below:

$$
\begin{aligned}
a_{6}=\frac{1}{11520 \pi^{4}}( & -23040 a_{0}-192 \pi^{2} a_{0}-\pi^{8} \omega^{2} a_{0} \\
& +11520 \pi a_{1}+32 \pi^{3} a_{1}+2 \pi^{7} \omega^{2} a_{1} \\
& -46080 a_{2}-5376 \pi^{2} a_{2}-32 \pi^{4} a_{2} \\
& +2 \pi^{8} \omega^{2} a_{2}+69120 \pi a_{3}+2304 \pi^{3} a_{3} \\
& \left.-59904 \pi^{2} a_{4}-768 \pi^{4} a_{4}+34560 \pi^{3} a_{5}\right)
\end{aligned}
$$

$$
\begin{aligned}
a_{7}=\frac{1}{80640 \pi^{3}}( & -768 a_{0}-16 \pi^{6} \omega^{2} a_{0}+28 \pi^{5} \omega^{2} a_{1} \\
& -\pi^{7} \omega^{2} a_{1}+1536 a_{2}-192 \pi^{2} a_{2} \\
& +36 \pi^{6} \omega^{2} a_{2}-2304 \pi a_{3}-96 \pi^{3} a_{3} \\
& +6 \pi^{7} \omega^{2} a_{3}+36864 a_{4}+3072 \pi^{2} a_{4} \\
& \left.-92160 \pi a_{5}-3840 \pi^{3} a_{5}+115200 \pi^{2} a_{6}\right)
\end{aligned}
$$

$$
\begin{aligned}
a_{8}=\frac{1}{322560 \pi^{4}} & \left(-768 a_{0}-112 \pi^{6} \omega^{2} a_{0}-384 \pi a_{1}\right. \\
& +168 \pi^{5} \omega^{2} a_{1}-16 \pi^{7} \omega^{2} a_{1}+1536 a_{2} \\
& -576 \pi^{2} a_{2}+280 \pi^{6} \omega^{2} a_{2}-\pi^{8} \omega^{2} a_{2} \\
& -2304 \pi a_{3}-672 \pi^{3} a_{3}+102 \pi^{7} \omega^{2} a_{3} \\
& +36864 a_{4}+4608 \pi^{2} a_{4}-192 \pi^{4} a_{4} \\
& +12 \pi^{8} \omega^{2} a_{4}-92160 \pi a_{5}-7680 \pi^{3} a_{5} \\
& +69120 \pi^{2} a_{6}-11520 \pi^{4} a_{6} \\
& +80640 \pi^{3} a_{7}
\end{aligned}
$$

$$
\begin{aligned}
a_{9}=\frac{1}{967680 \pi^{4}} & \left(-448 \pi^{5} \omega^{2} a_{0}-512 a_{1}+560 \pi^{4} \omega^{2} a_{1}\right. \\
& -112 \pi^{6} \omega^{2} a_{1}-1024 \pi a_{2}+1232 \pi^{5} \omega^{2} a_{2} \\
& -16 \pi^{7} \omega^{2} a_{2}-1920 \pi^{2} a_{3}+756 \pi^{6} \omega^{2} a_{3} \\
& -\pi^{8} \omega^{2} a_{3}+3072 \pi a_{4}-1408 \pi^{3} a_{4} \\
& +200 \pi^{7} \omega^{2} a_{4}-7680 \pi^{2} a_{5}-320 \pi^{4} a_{5} \\
& +20 \pi^{8} \omega^{2} a_{5}-92160 \pi a_{6}-46080 \pi^{3} a_{6} \\
& +322560 \pi^{2} a_{7}-26880 \pi^{4} a_{7} \\
& \left.-645120 \pi^{3} a_{8}\right)
\end{aligned}
$$

$$
\begin{aligned}
a_{10}=\frac{1}{2419200 \pi^{4}} & \left(-1120 \pi^{4} \omega^{2} a_{0}+1120 \pi^{3} \omega^{2} a_{1}\right. \\
& -448 \pi^{5} \omega^{2} a_{1}-320 a_{2}+3360 \pi^{4} \omega^{2} a_{2} \\
& -112 \pi^{6} \omega^{2} a_{2}-2688 \pi a_{3}+3192 \pi^{5} \omega^{2} a_{3} \\
& -16 \pi^{7} \omega^{2} a_{3}+1536 a_{4}-4032 \pi^{2} a_{4} \\
& +1456 \pi^{6} \omega^{2} a_{4}-\pi^{8} \omega^{2} a_{4}-3840 \pi a_{5} \\
& -2400 \pi^{3} a_{5}+330 \pi^{7} \omega^{2} a_{5}-46080 a_{6} \\
& -80640 \pi^{2} a_{6}-480 \pi^{4} a_{6}+30 \pi^{8} \omega^{2} a_{6} \\
& +161280 \pi a_{7}-134400 \pi^{3} a_{7} \\
& -322560 \pi^{2} a_{8}-53760 \pi^{4} a_{8} \\
& \left.-3386880 \pi^{3} a_{9}\right)
\end{aligned}
$$

In our computations, taking 175 terms for each $\psi_{k}(\omega, \theta)$ gave sufficient accuracy.

Applying the boundary conditions (25b) to Eq. (43) results in a system of six linear, homogeneous, algebraic equations; and the coefficient determinat equated to zero is the eigenvalue equation for $\omega$

$$
\begin{array}{|lcccc}
\psi_{0}(\pi / 4) & \psi_{1}(\pi / 4) & \psi_{2}(\pi / 4) & \psi_{3}(\pi / 4) & \psi_{4}(\pi / 4) \\
\psi_{0}(3 \pi / 4) & \psi_{1}(3 \pi / 4) & \psi_{2}(3 \pi / 4) & \psi_{3}(3 \pi / 4) & \psi_{4}(3 \pi / 4) \\
\psi_{0}{ }^{\prime}(\pi / 4) & \psi_{1}{ }^{\prime}(\pi / 4) & \psi_{2}{ }^{\prime}(\pi / 4) & \psi_{3}{ }^{\prime}(\pi / 4) & \psi_{4}{ }^{\prime}(\pi / 4) \\
\psi_{0}{ }^{\prime}(3 \pi / 4) & \psi_{1}{ }^{\prime}(3 \pi / 4) & \psi_{2}{ }^{\prime}(3 \pi / 4) & \psi_{3}{ }^{\prime}(3 \pi / 4) & \psi_{4}{ }^{\prime}(3 \pi / 4) \\
\psi_{0}{ }^{\prime \prime}(\pi / 4) & \psi_{1}{ }^{\prime \prime}(\pi / 4) & \psi_{2}{ }^{\prime \prime}(\pi / 4) & \psi_{3}{ }^{\prime \prime}(\pi / 4) & \psi_{4}{ }^{\prime \prime}(\pi / 4) \\
\psi_{0}{ }^{\prime \prime}(3 \pi / 4) & \psi_{1}{ }^{\prime \prime}(3 \pi / 4) & \psi_{2}{ }^{\prime \prime}(3 \pi / 4) & \psi_{3}{ }^{\prime \prime}(3 \pi / 4) & \psi_{4}{ }^{\prime \prime}(3 \pi / 4) \\
=0 & & & &
\end{array}
$$


Note that each entry in this determinat involves $\omega$ in a complicated way as can be seen from Eq. (43). We take $N=5$; the corresponding eigenvalues are

$$
\begin{aligned}
& \omega_{1}=\mp 9.12338967737348743757275069814 \\
& \omega_{2}=\mp 17.2406494706429733466236011265 \\
& \omega_{3}=\mp 31.4436893088065109131632954686
\end{aligned}
$$

\section{Results and Discussion}

Figs. 1 and 2 show the first five mode shapes for both u (normal displacement) and $\mathrm{W}$ (tangential displacement)

First mode shape for normal displacement.

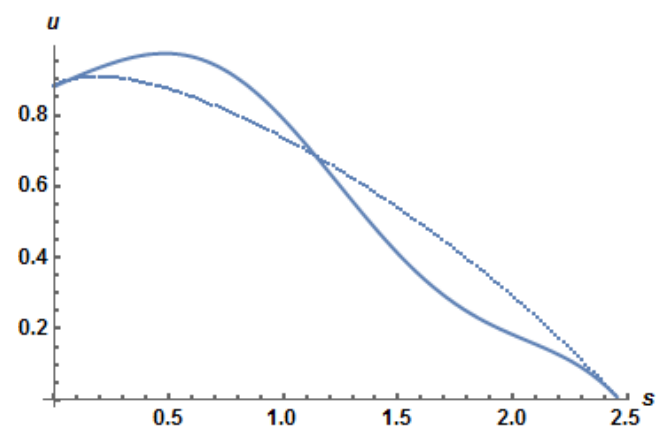

Third mode shape for normal displacement.

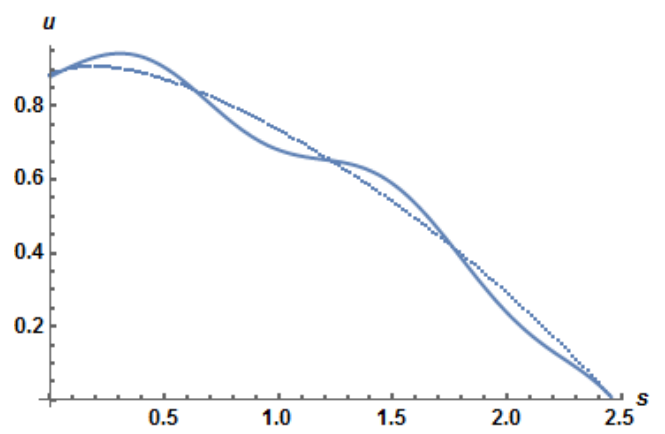

$$
\begin{gathered}
\omega_{4}=\mp 46.2998412670707832165432810622 \\
\omega_{5}=\mp 66.922713782870457545444711657
\end{gathered}
$$

superimposed on the unloaded shape of the beam axis.
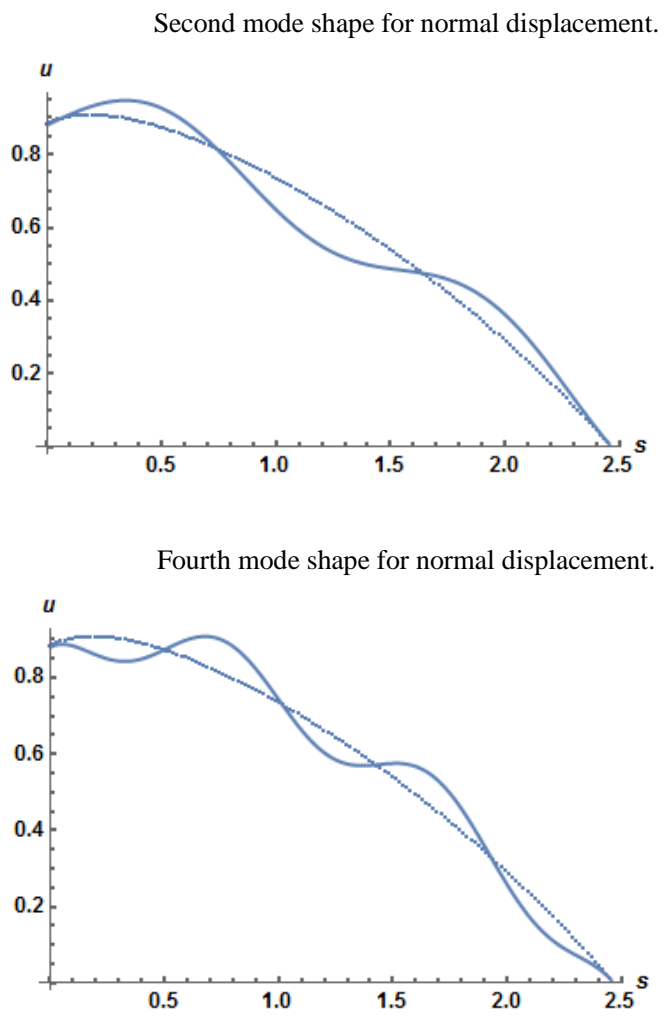

Fifth mode shape for normal displacement. 


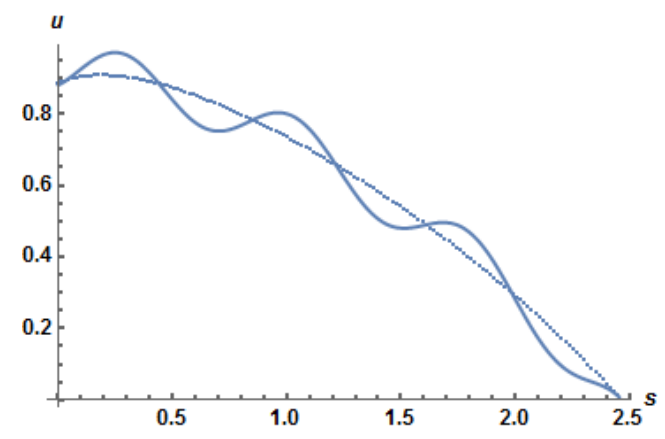

Figure 1. Mode shapes for normal displacement.
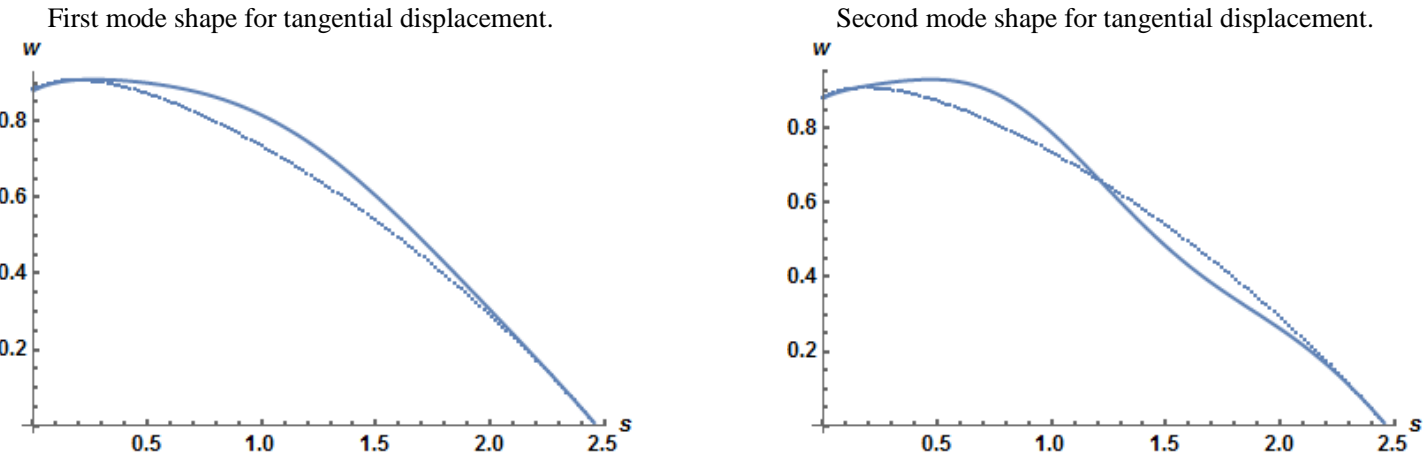

Third mode shape for tangential displacement.

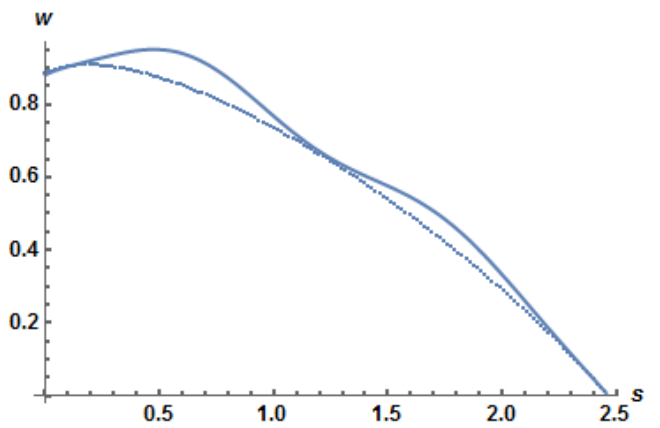

Fourth mode shape for tangential displacement.

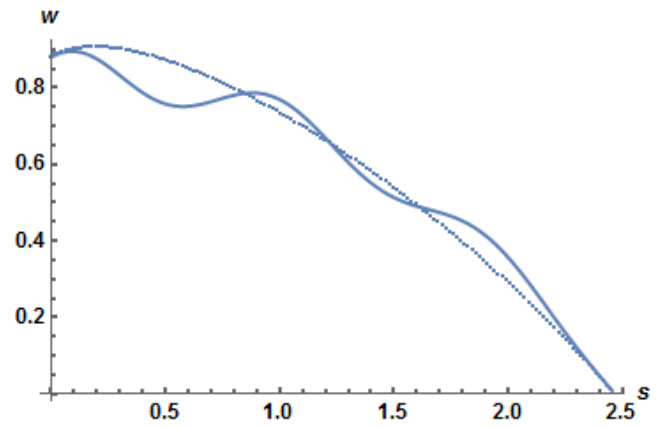

Fifth mode shape for tangential displacement.

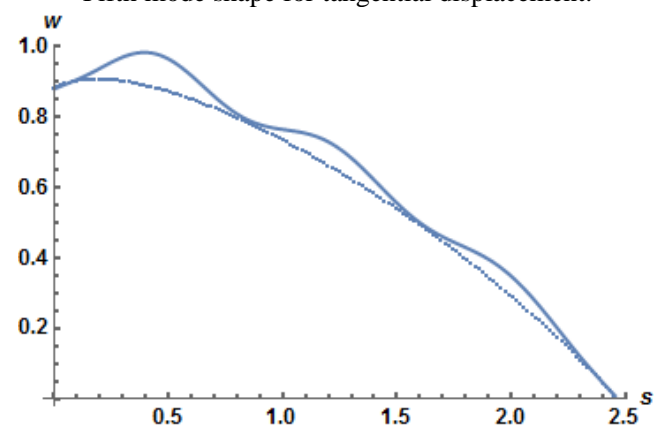

Fig. 2. Mode shapes for tangential displacement. 
We choose the non-dimensional velocity of the load as $\pi^{2} / 20$ so the load remains on the beam between $t=0$ and $t=5$. At $t=0$, the beam is not moving; therefore only the forced part of the solution of Eq. (34) is computed for $0 \leq t \leq 5$. After the load leaves the beam, only the homogeneous part of the solution is used with the shape and velocity of the beam for $t=5$ from the previous solution taken as initial conditions. Fig. 3 shows the total displacement, $\sqrt{u^{2}+w^{2}}$. The sign and direction of the total displacement can be inferred from the components $\mathrm{u}, \mathrm{w}$. Therefore, these figures show the actual shape the beam has at these given times. The non-dimensional force is taken as $F=250$.
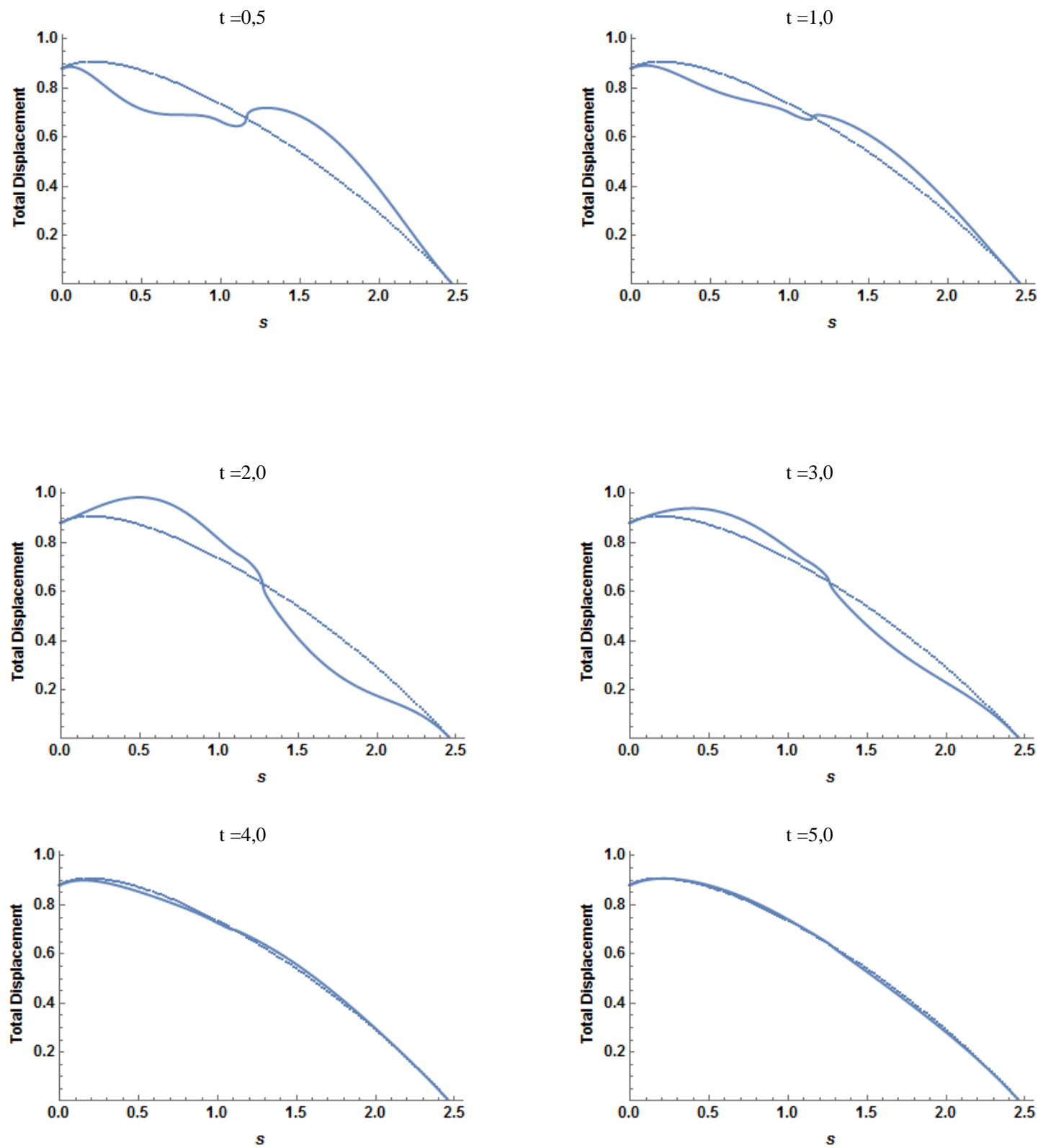

Fig. 3. Total Displacements at different times before the moving load leaves the beam.

At $t=5$ the load leaves the beam after which the total displacement (as explained above), multiplied by 5 is shown Fig. 3. 

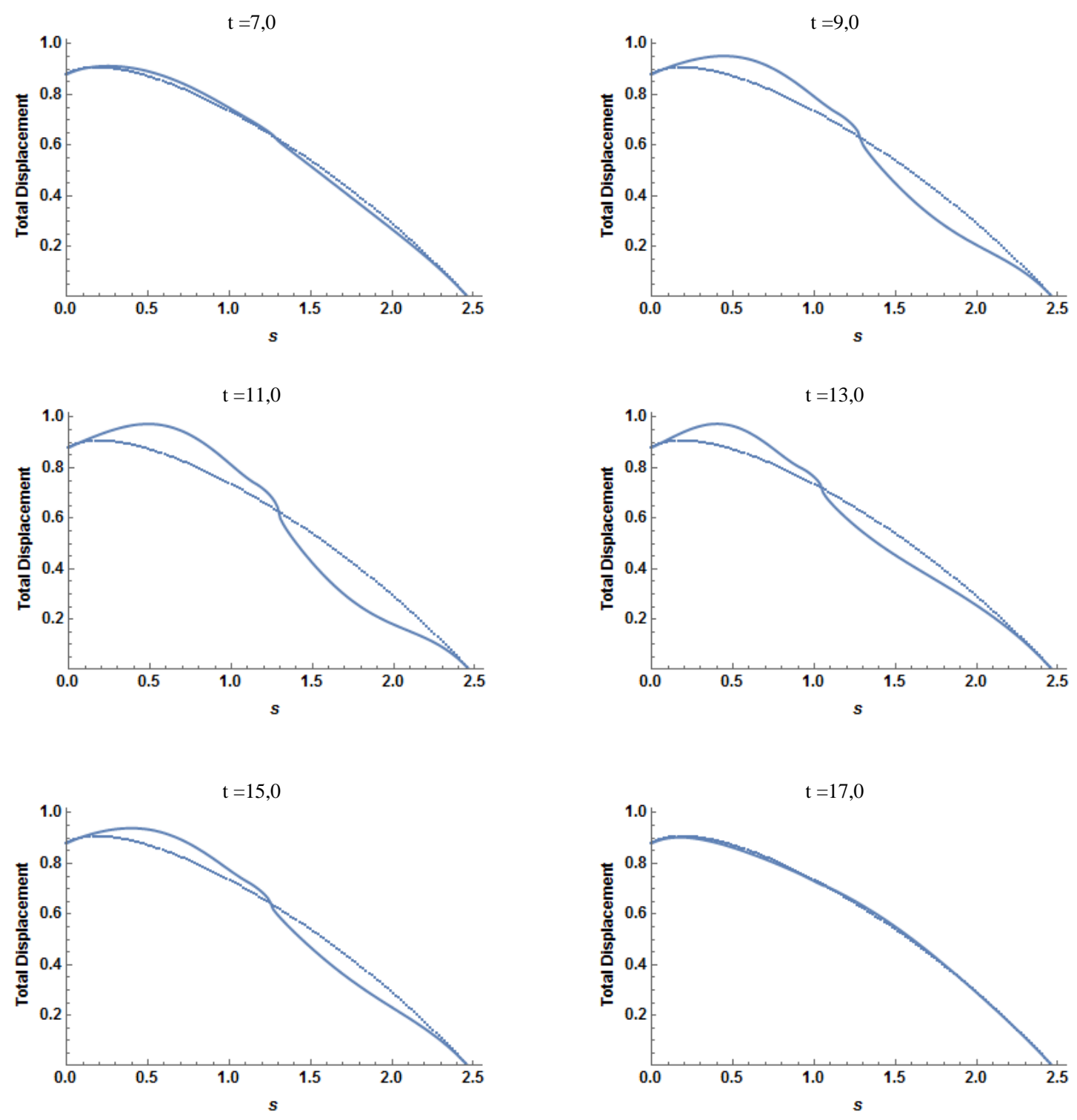

Fig. 4. Total Displacements at different times after the moving load leaves the beam.

Fig. 4. is free vibrations of the beam after the load leaves it. Comparing Fig. 4. ( $\mathrm{t}=15)$ and Fig. 4. $(\mathrm{t}=17)$ for example, the displacement is larger in Fig. 4. $(\mathrm{t}=15)$. This means the velocity would be larger in Fig. 4. $(\mathrm{t}=17)$ since the total energy is

\section{Conclusions and Recommendations}

We reported an analytic series solution for the response of a plane, arbitrarily curved beam subject to a moving load. The shape of the beam was computed both when the load is on the beam and after the load leaves the beam. Mode shapes were also computed and presented as part of the solution. Although the load was assumed to be always in the direction of the normal to the beam axis, loading in any direction in the plane can be considered.

It can be observed that the mode shapes for the normal displacement involve more points of inflection compared to the conserved. Looking at Fig. 3. the displacement is larger immediately after the load is on the beam. In this case the total energy is not conserved because of the force.

mode shapes of the tangential displacement. The simple reason for this is the relation between them, Eq. (5). The vibrations are more chaotic, local and have larger amplitude immediately following the entrance of the moving load on the beam from the boundary. As the load moves towards the other boundary, the vibrations become more ordered and less in amplitude, probably due to the effects of the moving load being felt at the other parts of the beam. After the load leaves the beam, the beam undergoes free, undamped vibrations since no damping effects are considered here. The vibrations seem to repeat at roughly 10 second-intervals. 


\section{References}

1. Love, A.E.H. (1944). A Treatise on the mathematical theory of elasticity.

2. Wu, J.S., Chiang, L.K. (2004). Dynamic analysis of an arch due to a moving load. J. Sound Vib. 269, 511-534.

3. Gulyayev, V.I., Tolbatov, E.Y. (2004). Dynamics of spiral tubes containing internal moving masses of boiling liquid. J. Sound Vib. 274, 233-248.

4. Wayou, A.N.Y., Tchoukuegno, R., Woafo, P. (2004). Nonlinear dynamics of an elastic beam under moving loads. J. Sound Vib. 273, 1101-1108.

5. Forbes, G.L., Randall, R.B. (2008). Resonance phenomena of an elastic ring under a moving load. J. Sound Vib. 318 , 991-1004.

6. Huang, J.L., Su, R.K.L., Lee, Y.Y., Chen, S.H. (2011). Nonlinear vibration of a curved beam under uniform base harmonic excitation with quadratic and cubic nonlinearities. J. Sound Vib. 330, 5151-5164.

7. Tufekci, E., Dogruer, O.Y. (2006). Out-of-plane free vibration of a circular arch with uniform cross-section: Exact solution. J. Sound Vib. 291, 525-538.

8. Yang, F., Sedaghati, R., Esmailzadeh, E. (2008). Free inplane vibration of general curved beams using finite element method. J. Sound Vib. 318, 850-867.

9. Ozturk, H. (2011). In-plane free vibration of a pre-stressed curved beam obtained from a large deflected cantilever beam. Finite Elem. Anal. Des. 47, 229-236.

10. Tolomeo, J.A. (2005). Bending of a simple beam to an optically accurate parabolic shape. Int. J. Solids Struct. 42, 1819-1830.

11. Lin, K.C., Hsieh, C.M. (2007). The closed form general solutions of 2-D curved laminated beams of variable curvatures. Compos. Struct. 79, 606-618.

12. Piovan, M.T., Cortinez, V.H. (2007). Mechanics of thinwalled curved beams made of composite materials, allowing for shear deformability. Thin Wall. Struct. 45, 759-789.

13. Wang, M., Liu, Y. (2013). Elasticity solutions for orthotropic functionally graded curved beams. Eur. J. Mech. A Solids 37, 8-16.

14. Lenci, S., Clementi, F. (2009). Simple mechanical model of curved beams by a 3D approach. J. Eng. Mech. 135(7), 597-613.

15. Lim, C.W. Wang, C.M., Kitipornchai, S. (1997). Timoshenko curved beam bending of Euler-Bernoulli solutions. Arch. Appl. Mech. 67, 179-190.

16. Luu, A.T., Kim, N.I., Lee, J. (2015). NURBS - based isogeometric vibration analysis of generally laminated deep curved beams with variable curvature. Compos. Struct. 119, 150-165.

17. Tseng, Y.P., Huang, C.S., Kao, M.S. (2000). In-plane vibration of laminated curved beams with variable curvature by dynamic stiffness analysis. Compos. Struct. 50, 103-114.

18. Krishnan, A., Suresh, Y.J. (1998). A simple cubic linear element for static and free vibration analyses of curved beams. Comput. Struct. 68, 473-489.

19. Kim, N.1., Seo, K.J., Kim, M.Y. (2003). Free vibration and spatial stability of non-symmetric thin-walled curved beams with variable curvatures. Int. J. Solids Struct. 40, 3107-3128.

20. Lee, B.K., Oh, S.J., Mo, J.M., Lee, T.E. (2008). Out-ofplane free vibrations of curved beams with variable curvature. J. Sound Vib. 318, 227-246.

21. Huang, C.S., Tseng, Y.P., Chang, S.H., Hung, C.L. (2000). Out-of-plane dynamic analysis of beams with arbitrarily varying curvature and cross-section by dynamic stiffness matrix method. Int. J. Solids Struct. 37, 495-513.

22. Huang, C.S., Tseng, Y.P., Leissa, A.W., Nieh, K.Y. (1998). An exact solution for in-plane vibrations of an arch having variable curvature and cross section. Int. J. Mech. Sci. 40(11), 1159-1173.

23. Sakman, L.E., Uzal, E. (2017). Exact solution for the vibrations of an arbitrary plane curved pipe conveying fluid. ZAMM-Z Angew Math Me. 97(4), 422-432. 